\title{
The weak magnetic field (WMF) enhances the stimulation of polymyxin $B$ sulfate (POL) on Vibrio qinghaiensis sp.-Q67
}

\author{
Ya-Qian Xu ${ }^{1,3}$, Kai Li ${ }^{1,2}$,Ze-Jun Wang ${ }^{1}$ and Shu-Shen Liu ${ }^{1,2,3^{*}}$ (1)
}

\begin{abstract}
Background: The weak magnetic field (WMF) can enhance the ability to remove target pollutants in wastewater, which drives us to consider whether WMF could give rise to the hormesis or not. In our previous study, it was found that polymyxin B sulfate (POL) can induce weak hormesis on Vibrio qinghaiensis sp.-Q67 (Q67). To this end, we set up four different WMF treatments during Q67 culture and POL exposure process, having no WMF in all cases (NW), adding WMF all the time (AW), exerting WMF only during the bacterial culture (BW), and exerting WMF only in POL exposure period (EW).
\end{abstract}

Results: It was shown that the concentration-response curves (CRCs) of POL in four WMF treatments at the exposure times of 6,9 , and $12 \mathrm{~h}$ are non-monotonic hormetic curves where the maximum stimulative effects $\left(E_{\text {min }}\right)$ of $P O L$ in BW and EW are obviously larger than those in AW and NW. The maximum $E_{\min }$ is $26.8 \%$ occurring in $\mathrm{EW}$ and $20.7 \%$ in BW at $6 \mathrm{~h}$, while the max $E_{\min }$ is $14.6 \%$ in NW at $9 \mathrm{~h}$, it means that stimulations of POL in BW and EW are earlier and stronger than those in NW. These findings first indicated that WMF can enhance the hormesis of POL.

Conclusions: This study showed that WMF as a key factor may influence the maximum stimulation effect of hormesis. The characteristic of biphasic (hormetic effect) challenges the traditional classical threshold model that is close to chemical risk assessment. But the mechanism of hormesis even now is inconclusive. WMF as a novelty and neglected factor has the potential to support the further development of hormesis mechanism.

Keywords: Heptapeptide antibiotic, Weak magnetic field, Time-dependent dose-response, Non-monotonic concentration-response, Least square support vector regression (LS-SVR)

\section{Background}

The interaction between external and internal environmental factor on living organisms has grown wide interest $[9,16,20]$. Till now, scientists have not reached a consensus on how living organisms reacting to this complicated circumstance, such as magnetic field, solar activity indices, temperature, humidity, atmospheric pressure, geomagnetic conditions, etc. $[4,33,58,61,66,72,76]$.

\footnotetext{
*Correspondence: ssliuhl@263.net

${ }^{1}$ Key Laboratory of Yangtze River Water Environment, Ministry of Education, College of Environmental Science and Engineering, Tongji University, Shanghai 200092, People's Republic of China

Full list of author information is available at the end of the article
}

Hence, model experiments make it possible to trace certain patterns of such interactions, which may both have a general physical content [66] and be of interest applications [58]. It was demonstrated that some chemicals could induce the hormesis in vitro by very low-dose environmental factors such as low-dose-rate gamma rays and $\mathrm{X}$-rays $[62,82]$.

Hormesis, a dose-response relationship characterized by a low-dose stimulation and a high-dose inhibition [11] has drawn increasing interests on dose-response studies, as it has either a harmful, beneficial or indifferent effect in an environment. In recent decades, a growing body of evidence has accumulated on hormetic effects of several chemicals for a number of biological endpoints 
of cellular systems and organisms [1, 11, 13, 18, 19, 57]. Hormetic effects were typically represented in graphs as an inverted U- or J-shaped dose-response, depending on the endpoint measured [9]. This dose-response represents an evolutionarily conserved process of adapting to changing environments, potentially beneficial responses to low doses of a stressor agent/condition [10] which would make the current ecosystem more balanced [15].

On the other hand, studies on the reaction of biological systems to magnetic fields (MF) are of great interest for not only fundamental science (ecology and biophysics) but also applied science, as some aquatic ecosystems become subject to electromagnetic pollution due to an increase in output of anthropogenic sources of low-frequency electromagnetic fields (EMF) [8]. Weak magnetic field (WMF) is of special interest among weak environmental factors that influence living systems, such as weak low-frequency EMF from electrical equipment. In terms of thermodynamics, the energy obtained in such action is insufficient to reach the thermal noise limit but results in clear repeatable bioeffects [33]. At present, the research on WMF in the field of environmental science (has focused on or) focus on water treatment technology. Application of zerovalent iron (ZVI) has been proved to be an environmentally friendly approach for heavy metal removal from water due to its low cost, simplicity in handling, and scalability in aquatic ecosystems [32, 39]. It has been proved that ZVI corrosion could be promoted by WMF over a wide $\mathrm{pH}$ range, resulting in more rapid release of some heavy metals $[37,63]$, such as the effect of WMF on metal (such as $\mathrm{Cu}[28,31], \mathrm{Sb}[35,70], \mathrm{Cr}$, As $[63,64]$, Se [37]) removal from water by ZVI. But the ultimate application of these technologies is oriented to the aquatic ecosystem, although not at present. Nevertheless, the mechanisms of how WMF and low-frequency EMF influence on living systems are seldom discussed as well as no commonly accepted models regarding to the biological effect $[7,45,64,68,71,80]$. Therefore, it is of great significance to explore whether WMF affects the doseresponse relationship of environmental pollutants and its toxic effects.

Polymyxin B sulfate (POL), an antibiotic widely used in the medical profession and the general public health, which is mainly used for the infections caused by sensitive bacteria and the urinary tract infection caused by pseudomonas aeruginosa, meningitis, sepsis, burn infection and skin mucosal infection, and so on [5, 23, 24, 34]. It was found that POL has weak time-dependent hormetic dose/concentration-response profile on Vibrio qinghaiensis sp.-Q67 (Q67) [25], which reminds us to think whether the WMF can influence the hormetic relationship of POL on Q67 or not. The main purpose of this paper is to examine the effect of WMF on the hormesis of POL by means of setting up different MF treatments in the time-dependent toxicity tests.

\section{Materials and methods Chemicals}

Polymyxin B sulfate was purchased from TRC (Canada, CAS: 1405-20-5, 95\% Purity). The stock solution concentration of POL was $1.521 \mathrm{E}-05 \mathrm{~mol} / \mathrm{L}$. All solutions were prepared with Milli-Q water and stored in darkness at $4{ }^{\circ} \mathrm{C}$ before the test. The molecular structure of POL is shown in Fig. 1.

\section{Q67 culture}

The luminescent bacterium Q67 (Beijing Hamamatsu Corp., Ltd., Beijing, China) was incubated in liquid culture medium with a shaking speed of $120 \mathrm{rpm}$ at $22 \pm 1{ }^{\circ} \mathrm{C}$ for 9-10 h, and the bacteria in the logarithmic growth phase were used in experiments [36, 41, 78, 84]. Then, the culture medium containing Q67 was mixed with an equal amount of the twofold concentrated medium [67, 73]. Based on the growth curve in the mixed culture medium, Q67 was further incubated for about 40-60 min.

\section{Time-dependent toxicity test}

A time-dependent microplate toxicity analysis (t-MTA) is exactly the same as that in the previous works $[55,56,81]$. For the test chemicals, 12 diluted concentration series in 3 parallels and 12 controls were arranged in the white 96-well standard opaque plates with 12 rows and 8 columns (Corning Corp.). To avoid possible edge effects, the first and eighth rows, together with the first column and the last column (36 wells in total) were filled with $200 \mu \mathrm{L}$ distilled water. For the remaining 60 wells, 24 wells from the second, sixth, seventh, and eleventh columns were treated as controls. The rest of the plate (36 wells) was arranged with 12 concentration series of the toxicants, and it was divided into 2 sections (a left one and a right one), both of which had 6 rows and 3 columns. The left section was arranged with 6 higher concentration series,

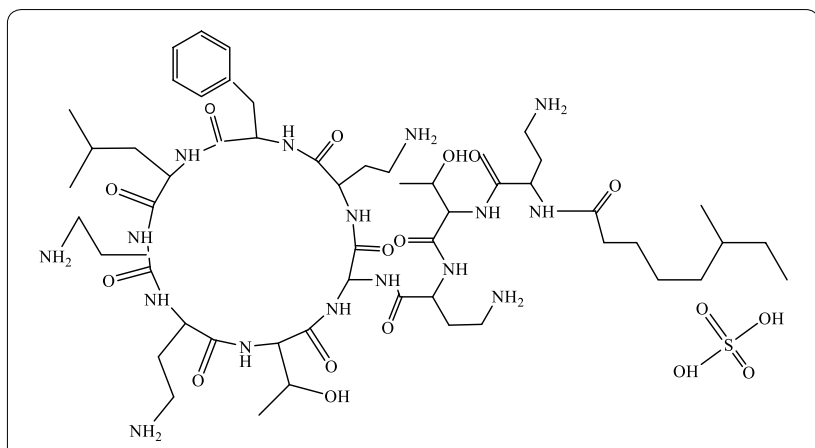

Fig. 1 The molecular structure of polymyxin B sulfate (POL) [25] 
and each concentration used 1 row with 3 wells as parallel. The right section was arranged with 6 lower concentration series. Each of the 60 wells contained $100 \mu \mathrm{L}$ toxicants or distilled water (as controls). The prepared bacteria were added into the 60 wells to make the final test volume be $200 \mu \mathrm{L}$ in each well [81].

To evaluate the toxic effects of POL at different times and various concentrations, the relative light unit (RLU) of every well was determined on the Power-Ware microplate spectrophotometer (American BIO-TEK Company) at $22 \pm 1{ }^{\circ} \mathrm{C}$. During exposure, readings were taken at $0.25,3,6,9$ and $12 \mathrm{~h}$. Inhibition ratio of bioluminescence was used to characterize the toxicity or toxic effect, noted as $E$,

$$
E=\frac{I_{0}-I}{I_{0}}
$$

where $I_{0}$ is the average RLU of Q67 exposed to the control groups and $I$ the average RLU of Q67 exposed to the experimental groups.

For the monotonic concentration-effect $(C-E)$ data in different exposure times, the concentration-effect/ response curves (CRCs) are modeled by the nonlinear least squares fit [42] and for the non-monotonic $C-E$ data, the CRCs are fitted by the least squares support vector regression (LS-SVR) procedure [53] or a method for the fitting and prediction of J- and S-shaped concentrationresponse curves (JSFit) program [69]. The goodness of fit is expressed as the determination coefficient $\left(R^{2}\right)$. The analysis of variance (ANOVA) (Origin Pro 7.5, Origin Lab Corp., USA) was carried out among the results from independent experiments, and the significance levels of $0.05(P<0.05)$ were considered statistically significant.

\section{Weak magnetic field treatment}

The static, nonuniform magnetic field was supplied by a cuboid permanent magnet of $40 \times 20 \times 3 \mathrm{~mm}^{3}$. Figure 2 presents a diagrammatic sketch of experimental device in the process of bacterial culture (Fig. 2a) and POL exposure (Fig. 2b). In the process of bacterial culture, six permanent magnets were set at the front (one magnet), back (one), both sides (two), and bottom (two) of the beaker flask of $100 \mathrm{~mL}$ in which there is $33 \mathrm{~mL}$ bacteria suspension, respectively (Fig. 2a). In the process of chemical exposure, six permanent magnets were also set at the top (two magnets), bottom (two) and both sides (two) of the microplates, respectively (Fig. 2b). The maximum magnetic intensity was determined to be $\sim 20 \mathrm{mT}$ with six permanent magnets.

In this study, four WMF experimental groups, one without WMF (NW) in all cases, one with WMF (AW) in all cases, one with WMF only during the bacterial culture
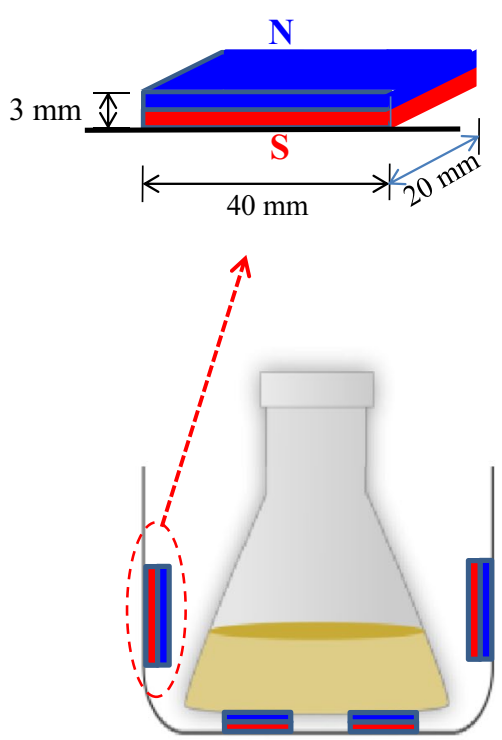

$33 \mathrm{~mL}$ bacterial suspension in $100 \mathrm{~mL}$ beaker flask

a Culture with WMF
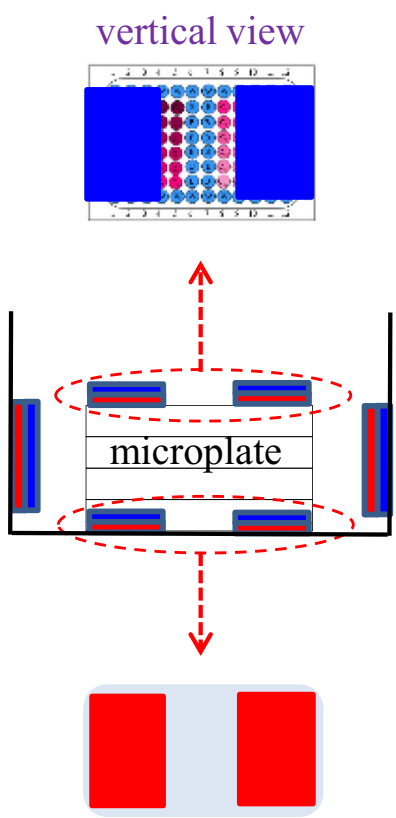

Bottom view

b Exposure with WMF

Fig. 2 The diagrammatic sketch of a laboratory-scale device with WMF where a illustrates the size and layout of the permanent magnets in exposure duration and $\mathbf{b}$ depicts the layout of the permanent magnets during the Q67 culture duration 
period (BW), and one with WMF only in POL exposure period $(\mathrm{EW})$, were created.

\section{Results}

\section{Concentration-response relationships of POL}

The concentration-inhibition data of POL in four WMF treatments at different time points can be fitted by the LS-SVR [53]. The fitted concentration-response curves (CRCs) were subsequently used to calculate three main characterization parameters of the hormetic concentration-response curve (hCRC) such as the median effective concentrations $\left(\mathrm{EC}_{50}\right)$, the minimum inhibitory effect $\left(E_{\min }\right)$ and its corresponding concentration $\left(\mathrm{EC}_{\min }\right)$. The fitted regularization parameter (gam), kernel function parameter (sig2), determination coefficient $\left(R^{2}\right)$, and three characterization parameters, $\mathrm{EC}_{50}, E_{\min }$, and $\mathrm{EC}_{\text {min }}$ are listed in Table 1.

From Table $1, R^{2}$ is higher than 0.980 (one exception), which indicates that all J-shaped hCRCs are well fitted by LS-SVR procedure. Taking $\mathrm{EC}_{50}$ as a toxicity index, apart from the toxicity of POL in four WMF treatments at the first time point $(0.25 \mathrm{~h})$ is less, those at the other four time points are almost the same. $\mathrm{EC}_{\min }$ in all WMF treatments at all time points are around $1.00 \mathrm{E}-07 \mathrm{~mol} / \mathrm{L}$, which implies that both the WMF and exposure time do not alter the minimum inhibitory effective concentration.
However, both WMF and time can alter the maximum simulative effect $\left(E_{\min }\right)$ of POL on Q67. The concentration-inhibition profiles of POL in four WMF treatments at different time points are shown in the three-dimensional (3D) CRCs (Fig. 3).

In each WMF treatment (Fig. 3), five CRCs of POL at different exposure times are different from each other, which depict the time-dependence of CRCs again. Obviously, the latter three CRCs in BW and EW groups show more significant J-shape nature than those in NW and AW, their simulative effect being greater, which demonstrates that the WMF indeed enhance the hormesis, significantly improving the simulative effect of POL.

\section{Hormetic effects of POL}

Polymyxin B sulfate shows hCRCs on Q67 at 0.25, 3, 6, 9, and $12 \mathrm{~h}$ in all WMF groups. $E_{\min }$ is from $3.6 \%$ (at $3 \mathrm{~h}$ in $\mathrm{AW}$ ) to $26.8 \%$ (at $6 \mathrm{~h}$ in $\mathrm{BW}$ ) and $\mathrm{EC}_{\text {min }}$ from 7.508E-08 (at $0.25 \mathrm{~h}$ in NW) to $7.286 \mathrm{E}-07$ (at $12 \mathrm{~h}$ in BW) (Table 1). Notably, EW owns the maximum stimulative effect at $6 \mathrm{~h}, E_{\min }=26.8 \%$; BW has the maximum stimulative effect at $6 \mathrm{~h}, E_{\min }=20.7 \%$; while $E_{\min }$ of NW is $14.6 \%$ at $9 \mathrm{~h}$ (Fig. 4a). That is, stimulations of POL in BW and EW are earlier and stronger than those in NW. However, the

Table 1 The fitting parameters (gam, sig2 and $R^{2}$ ) of various LS-SVR models and three characterization parameters $\left(\mathrm{EC}_{50}\right.$ $E_{\min }$, and $E C_{\min }$ ) from the CRCs of POL in four WMF groups at five times

\begin{tabular}{|c|c|c|c|c|c|c|c|}
\hline WMF group & Time (h) & gam & $\operatorname{sig} 2$ & $R^{2}$ & $\mathrm{EC}_{50}(\mathrm{~mol} / \mathrm{L})$ & $E_{\min }(\%)^{a}$ & $\mathrm{EC}_{\min }(\mathrm{mol} / \mathrm{L})$ \\
\hline \multirow[t]{5}{*}{ NW } & 0.25 & 91 & 0.5 & 0.9981 & $5.629 \mathrm{E}-06$ & -7.3 & $7.508 \mathrm{E}-08$ \\
\hline & 3 & 91 & 0.3 & 0.9988 & $3.000 E-06$ & -7.8 & $8.569 \mathrm{E}-08$ \\
\hline & 6 & 71 & 0.3 & 0.9976 & $2.954 \mathrm{E}-06$ & -11.2 & $1.564 \mathrm{E}-07$ \\
\hline & 9 & 91 & 0.4 & 0.9921 & $3.165 E-06$ & -14.6 & 4.409E-07 \\
\hline & 12 & 91 & 0.3 & 0.9967 & $3.314 \mathrm{E}-06$ & -13.0 & $6.272 \mathrm{E}-07$ \\
\hline \multirow[t]{5}{*}{ AW } & 0.25 & 91 & 0.4 & 0.9973 & $4.791 \mathrm{E}-06$ & -4.4 & $1.330 \mathrm{E}-07$ \\
\hline & 3 & 31 & 0.3 & 0.9932 & $2.887 \mathrm{E}-06$ & -3.6 & $4.509 \mathrm{E}-07$ \\
\hline & 6 & 21 & 0.4 & 0.9822 & $3.239 \mathrm{E}-06$ & -8.7 & $4.705 \mathrm{E}-07$ \\
\hline & 9 & 41 & 0.3 & 0.9945 & $3.551 \mathrm{E}-06$ & -14.0 & 7.109E-07 \\
\hline & 12 & 91 & 0.2 & 0.9994 & $3.470 \mathrm{E}-06$ & -15.6 & $6.550 \mathrm{E}-07$ \\
\hline \multirow[t]{5}{*}{ BW } & 0.25 & 91 & 0.5 & 0.9921 & $5.984 \mathrm{E}-06$ & -6.0 & $1.152 \mathrm{E}-07$ \\
\hline & 3 & 91 & 0.2 & 0.9976 & $3.551 \mathrm{E}-06$ & -7.8 & $6.850 \mathrm{E}-07$ \\
\hline & 6 & 81 & 0.2 & 0.9992 & $3.344 \mathrm{E}-06$ & -20.7 & $3.609 \mathrm{E}-07$ \\
\hline & 9 & 31 & 0.2 & 0.9869 & $4.038 \mathrm{E}-06$ & -19.6 & $6.055 \mathrm{E}-07$ \\
\hline & 12 & 21 & 0.2 & 0.9893 & $3.805 \mathrm{E}-06$ & -18.0 & $7.286 \mathrm{E}-07$ \\
\hline \multirow[t]{5}{*}{ EW } & 0.25 & 91 & 0.6 & 0.9986 & $5.501 \mathrm{E}-06$ & -5.0 & $3.484 \mathrm{E}-07$ \\
\hline & 3 & 90 & 0.3 & 0.9978 & $2.821 \mathrm{E}-06$ & -9.4 & $3.838 \mathrm{E}-07$ \\
\hline & 6 & 91 & 0.3 & 0.9975 & $3.239 \mathrm{E}-06$ & -26.8 & $3.338 \mathrm{E}-07$ \\
\hline & 9 & 91 & 0.4 & 0.9979 & $3.471 \mathrm{E}-06$ & -17.8 & $5.686 \mathrm{E}-07$ \\
\hline & 12 & 41 & 1 & 0.9784 & $3.023 \mathrm{E}-06$ & -23.6 & $4.991 \mathrm{E}-07$ \\
\hline
\end{tabular}

\footnotetext{
${ }^{a} E_{\min }$ refers to the minimum inhibitory effect or the maximum stimulative effect
} 

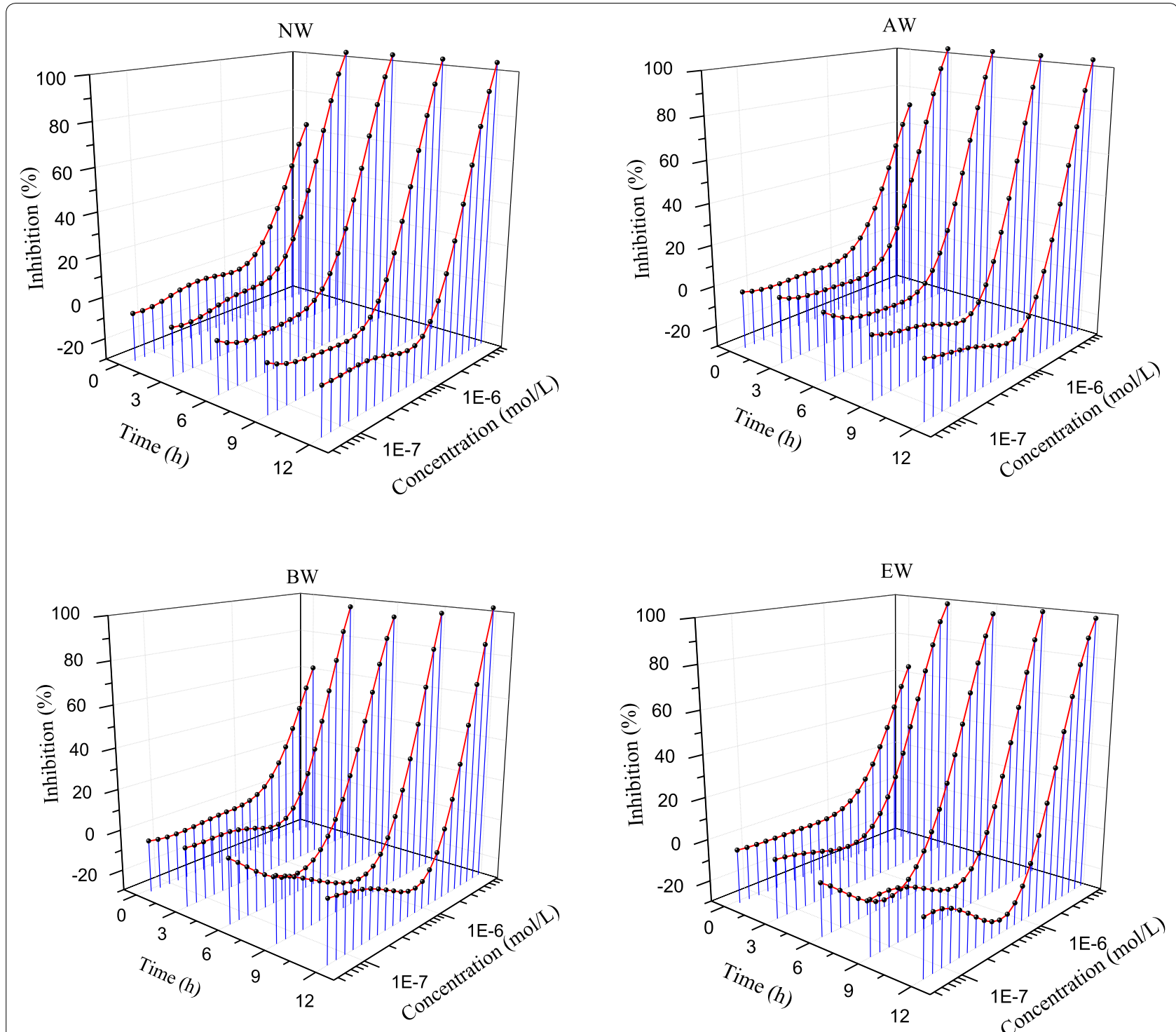

Fig. 3 The three-dimensional (3D) concentration-response curves of POL at five exposure times $(0.25$ h, 3 h, 6 h, 9 h, and 12 h) in four WMF groups (NW, AW, BW, and EW)

stimulative effect enhanced by weak magnetic field did not occur in AW (Fig. 4b).

The $E_{\min }$ of POL to Q67 in BW and EW are significantly larger than those in NW and AW $(P<0.05)$ at 6 , $9,12 \mathrm{~h}$. The $E_{\min }$ of POL in EW and BW are, respectively, $26.8 \%$ and $20.7 \%$ at $6 \mathrm{~h}$, being $183.6 \%$ and $141.8 \%$ of the maximum stimulative effect in NW at $9 \mathrm{~h}(14.6 \%)$.

\section{Discussion}

\section{Hormesis, ordinary or not?}

Figure 1 presents the time-dependent hormesis of POL on Q67. Fan et al. [25] and Liu et al. [40] found that some antibiotics did not have hormetic CRCs on Q67.
However, some ionic liquids and personal care products showed hCRCs on Q67 [55, 74, 75], implying that the different model organisms and different toxicity endpoint may result in different CRCs. For example, de Vasconcelos et al. [21] reported that some pharmaceuticals showed hCRCs on Vibrio fischeri and Desmodesmus subspicatus at low concentrations. Radak et al. [57] observed that physical exercise could evoke the hormetic curve response by the organism. Zhu et al. [85] found that some imidazolium-based ionic liquids exhibited hCRCs when took the lifespan of Caenorhabditis elegans as the toxicity endpoint. Many ionic liquids showed hormesis on different organisms, such as anaerobic Clostridium sp. and 

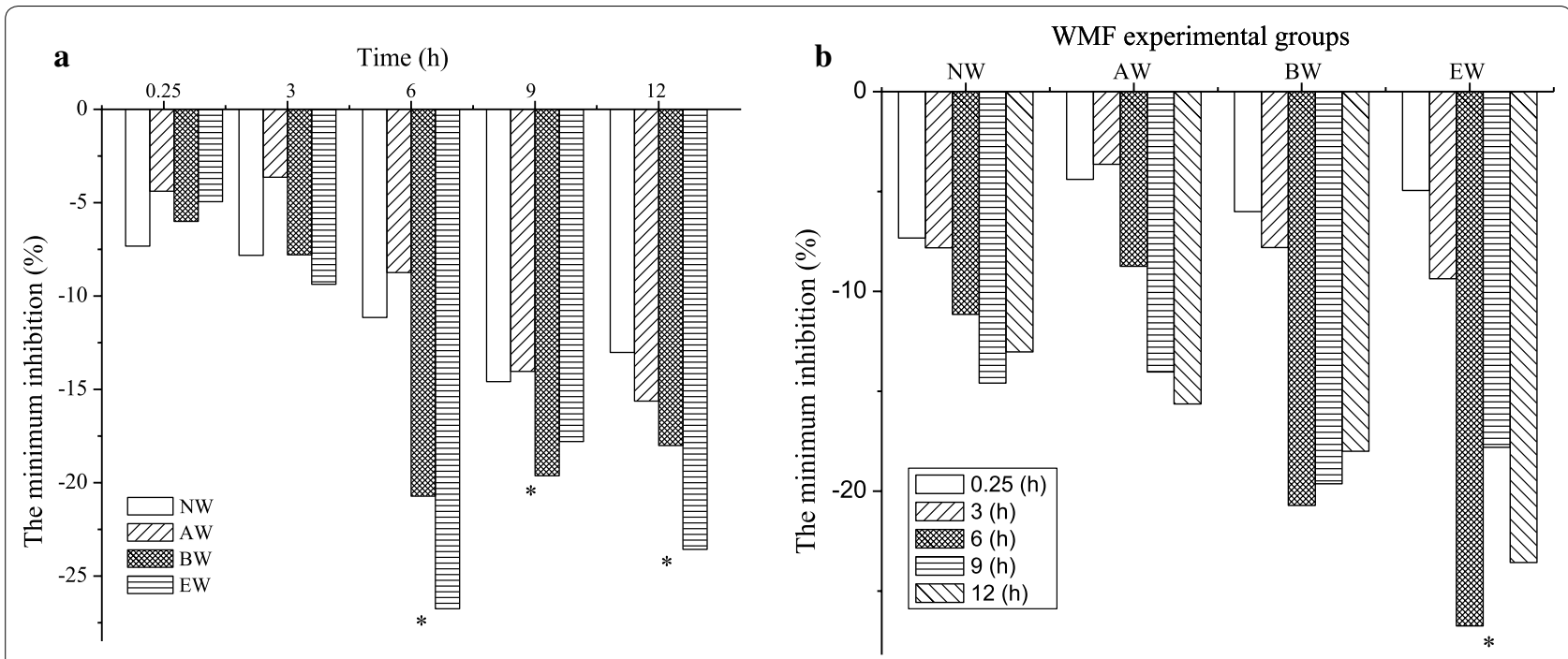

Fig. 4 The minimum inhibition ratios of different WMF groups (NW, AW, BW, and EW) at the same time (a) and different times $(0.25,3,6,9$, and $12 \mathrm{~h})$ in the same WMF group (b)

aerobic Pseudomonas putida [47], microalga Scenedesmus quadricauda [22], and firefly luciferase [27].

More recent pieces of the literature have demonstrated that hormetic effects occurred widespread with relatively high generalizability, reproducibility, frequency, and with a solid mechanistic foundations [12, 17]. Experiments showed that the damage and death of Q67 caused by gamma-ray radiation, which is the important reason for the inhibition of Q67 luminescence intensity [79]. However, the hormesis of Q67 occurred with low-dose gamma radiation [83]. Thus, hormesis is a common phenomenon. It could be found with different model organisms and different toxicity endpoints under different experimental conditions.

\section{The omission of weak environmental factors-WMF}

Table 1 shows that the toxicity of POL is basically unchanged with exposure time (except $0.25 \mathrm{~h}$ ), but the intensity of stimulative effect increases significantly by WMF (more negative). Obviously, WFM, as a weak environmental factor, plays an important role in the enhancement of stimulative effect.

In general, any changes in the experimental conditions will have an impact on organisms. The report provides evidence that the greater number of concentrations employed in the low-dose zone, the better the characterization of the hormetic stimulatory response [14]. External factors, i.e. environmental parameters, are beyond the control of the experimenter (magnetic field, solar activity indices, temperature, humidity, atmospheric pressure, geomagnetic conditions, etc.), while internal factors are genetic drift, instability of genomic transposable elements, as well as other aspects of genetic variation in populations, which are also practically uncontrollable [30]. Some scholars found that power-frequency electric and magnetic fields may cause interference with cardiac pacemakers [52, 77]. Krylov et al. [33] found that developing parthenogenetic eggs of Daphnia magna exposed to a number of low-frequency electromagnetic fields with indicated parameters have shown accelerated rates of embryonic development. Izmaylov et al. [30] have demonstrated that atmospheric pressure and geomagnetic activity are significantly correlated with the parameters of lifespan distributions in Drosophila melanogaster laboratory populations.

More and importantly, the report showed that neuronal activity might be affected by magnetogenetics, such as the direction of the magnetic field and the external magnetic field applied [43]; while a magnetic protein biocompass is well created, which is a protein complex and may form the basis of magnetoreception in animals, and may lead to applications across multiple fields [54]. Hence, there may be a type of magnetoreceptor in Q67, and the magnetoreceptor may activate the bioluminescence signal pathway and show stimulate effect. But we have no idea if POL could be as a bridge of this activation due to the WMF is never considered in other chemicals that show hormetic effects.

\section{Enhanced hormesis of POL}

Weak magnetic field is of special interest among weak environmental factors that influence living systems. Energy of such action is insufficient to exceed thermal noise limit in terms of thermodynamics, but clearly 
repeatable bioeffects take place nevertheless [33]. However, certain mechanisms of WMF's influence on living systems are actively discussed at present, and there are still no commonly accepted models of biological effect of this factor $[6,7,26,51,65]$.

With the WMF treatment, it is obvious that the stimulative effect of POL increased (in BW and EW). However, there was no significant difference between the AW and NW. It means that different periods of Q67 with the same WMF treatment (especially BW and EW) have different hormesis enhancement, but the same periods of Q67 with different WMF treatment (AW and NW) have no significant difference hormesis enhancement.

At the same time point $(6 \mathrm{~h})$, the maximum stimulation $(26.8 \%)$ occurs in EW, while the second maximum stimulation $(20.7 \%)$ occurs in BW. The hormesis of POL enhanced significantly by WMF. In animal model systems, it has been reported that WMF generally stimulated ornithine decarboxylase activity and cell proliferation $[38,46]$.

The increase of inflexibility caused by the large ring structure of POL (20-member ring of heptapeptide [24]) may lead to some directional action (such as permeability changes [44]) during the WMF treatment. The possible mechanisms underlying the contribution of these environmental factors about the hormesis need further investigation. Based on the view of quantum mechanics, "more is different", the complexity of the relevant mechanism will be beyond imagination [2].

\section{Different periods by WMF treatment}

Four experimental groups were constructed with different WMF treatments, including blank control and positive control. NW is blank control, AW is positive control, BW and EW are experimental groups. Research has continuously shown that it usually has a different toxic effect of chemicals on organism during different periods [3, 29, $48,50,59]$.

By comparing the results in AW, BW and EW with that in NW, hormesis enhancement is obtained during different WMF treatments. Q67 was further grown in the liquid medium when maintained for another $12 \mathrm{~h}$ to reach the logarithmic phase (BW), during this time, WMF may affect the metabolic activity of cells, leading more sensitive cell activities. This process is similar to domestication. Moreover, quorum sensing [49] signal pathway is modified to clarify the phenomenon when Q67 was exposed to POL in the toxicity test. However, EW may not necessarily so. It is likely that WMF has a protective effect on Q67 when exposing to low concentration of POL, thus an amplified direct stimulation [60] is expected to aim at protection. Because of little literature on this field, the true rule is still unclear, and it is difficult to explore the relevant mechanisms. It needs further investigation.

\section{Conclusions}

Taking POL as object chemical and Q67 as target organism by the method of $\mathrm{t}$-MTA, with four different WMF treatments (NW, AW, BW, and EW), the results showed that with the treatment of $\mathrm{BW}$ and $\mathrm{EW}$ the $E_{\min }$ of POL significant increased compared that of $\mathrm{NW}$, it indicated that different growth period of Q67 with the same WMF treatment has significant hormesis enhancement. In contrast, the $E_{\min }$ of AW has no significant difference with that of NW, it suggested that the same growth period of Q67 with different WMF have no significant hormesis enhancement. These findings prove that WMF as a neglectable factor, it actually and awfully enhanced the hormesis of POL on Q67. WMF as a complex factor during the organism growth stage, it may help unravel drivers of mechanisms of inhibition and stimulation.

\begin{abstract}
Abbreviations
WMF: weak magnetic field; POL: polymyxin B sulfate; Q67: Vibrio qinghaiensis sp.-Q67; NW: having no WMF in all cases; AW: adding WMF all the time; BW: exerting WMF only during the bacterial culture; EW: exerting WMF only in POL exposure period; $C R C s$ : the concentration-response curves; $E_{\min }$ : the maximum stimulative effects or the minimum inhibitory effect; $\mathrm{EC}_{\min }$ : the corresponding concentration of $E_{\text {min }}$ iLS-SVR: least square support vector regression; EMF: electromagnetic fields; ZVI: zerovalent iron; t-MTA: time-dependent microplate toxicity analysis; RLU: relative light unit; C-E: concentration-effect; JSFit: a method for the fitting and prediction of J- and S-shaped concentration-response curves; hCRC: the hormetic concentration-response curve; $\mathrm{EC}_{50}$ : the median effective concentrations; ANOVA: analysis of variance; gam: the fitted regularization parameter; sig2: Kernel function parameter; $R^{2}$ : determination coefficient; 3D: three-dimensional.
\end{abstract}

\section{Acknowledgements}

We are thankful to the National Natural Science Foundation of China (No. 21976139, No. 21677113, and No. 21437004) for their financial support.

\section{Authors' contributions}

YQX conceived and designed the experiment, determined the toxicity, analyzed the data, and wrote the paper. $\mathrm{KL}$ examined the language of the manuscript and supported table and figure analysis. SSL participated and supported with suggestions before and during the study. ZJW modified the calculation program. All authors read and approved the final manuscript.

\section{Funding}

The National Natural Science Foundation of China (No. 21976139, No. 21677113, and No. 21437004).

Availability of data and materials

The authors declare that all data supporting the findings of this study are available in the article and its additional information files.

\section{Ethics approval and consent to participate}

Not applicable.

Consent for publication

Not applicable.

Competing interests

The authors declare that they have no competing interests. 


\section{Author details}

${ }^{1}$ Key Laboratory of Yangtze River Water Environment, Ministry of Education, College of Environmental Science and Engineering, Tongji University, Shanghai 200092, People's Republic of China. ${ }^{2}$ State Key Laboratory of Pollution Control and Resource Reuse, College of Environmental Science and Engineering, Tongji University, Shanghai 200092, People's Republic of China. ${ }^{3}$ Shanghai Institute of Pollution Control and Ecological Security, Shanghai 200092, People's Republic of China.

Received: 11 October 2019 Accepted: 22 January 2020

Published online: 04 February 2020

\section{References}

1. Abbas T, Nadeem MA, Tanveer A, Zohaib A (2016) Low doses of fenoxaprop-p-ethyl cause hormesis in littleseed canarygrass and wild oat. Planta Daninha 34(3):527-533

2. Anderson PW (1972) More is different: broken symmetry and the nature of the hierarchical structure of science. Science 177(4047):393-396

3. Asih AYP, Irawan B, Soegianto A (2013) Effect of copper on survival, osmoregulation, and gill structures of freshwater prawn (Macrobrachium rosenbergii, de Man) at different development stages. Marine Freshw Behav Physiol 46(2):75-88

4. Belyavskaya NA (2004) Biological effects due to weak magnetic field on plants. Adv Space Res 34(7):1566-1574

5. Bergen PJ, Landersdorfer CB, Zhang J, Zhao M, Lee HJ, Nation RL, Li J (2012) Pharmacokinetics and pharmacodynamics of 'old' polymyxins: what is new? Diagn Microbiol Infect Dis 74(3):213-223

6. Binghy VN (1997) Interference of ion quantum states within a protein explains weak magnetic field's effect on biosystems. Electro- Magnetobiol 16(3):203-214

7. Binhi VN, Savin AV (2003) Effects of weak magnetic fields on biological systems: physical aspects. Phys Uspekhi 46(3):259-291

8. Bochert R, Zettler ML (2004) Long-term exposure of several marine benthic animals to static magnetic fields. Bioelectromagnetics 25(7):498-502

9. Calabrese EJ (2004) Hormesis: a revolution in toxicology, risk assessment and medicine-Re-framing the dose-response relationship. EMBO Rep 5:S37-\$40

10. Calabrese EJ (2008) Hormesis and medicine. Br J Clin Pharmacol 66(5):594-617

11. Calabrese EJ (2008) Hormesis: why it is important to toxicology and toxicologists. Environ Toxicol Chem 27(7):1451-1474

12. Calabrese EJ (2010) Hormesis is central to toxicology, pharmacology and risk assessment. Hum Exp Toxicol 29(4):249-261

13. Calabrese EJ (2017) Hormesis commonly observed in the assessment of aneuploidy in yeast. Environ Pollut 225:713-728

14. Calabrese EJ, Agathokleous E, Kozumbo WJ, Stanek EJ, Leonard D (2019) Estimating the range of the maximum hormetic stimulatory response. Environ Res 170:337-343

15. Calabrese EJ, Bachmann KA, Bailer AJ, Bolger PM, Borak J, Cai L, Cedergreen N, Cherian MG, Chiueh CC, Clarkson TW, Cook RR, Diamond DM, Doolittle DJ, Dorato MA, Duke SO, Feinendegen L, Gardner DE, Hart RW, Hastings KL, Hayes AW, Hoffmann GR, Ives JA, Jaworowski Z, Johnson TE, Jonas WB, Kaminski NE, Keller JG, Klaunig JE, Knudsen TB, Kozumbo WJ, Lettleri T, Liu S-Z, Maisseu A, Maynard KI, Masoro EJ, McClellan RO, Mehendale HM, Mothersill C, Newlin DB, Nigg HN, Oehme FW, Phalen RF, Philbert MA, Rattan SIS, Riviere JE, Rodricks J, Sapolsky RM, Scott BR, Seymour C, Sinclair DA, Smith-Sonneborn J, Snow ET, Spear L, Stevenson DE, Thomas Y, Tubiana M, Williams GM, Mattson MP (2007) Biological stress response terminology: integrating the concepts of adaptive response and preconditioning stress within a hormetic dose-response framework. Toxicol Appl Pharmacol 222(1):122-128

16. Calabrese EJ, Baldwin LA (2000) Tales of two similar hypotheses: the rise and fall of chemical and radiation hormesis. Hum Exp Toxicol 19(1):85-97

17. Calabrese EJ, Stanek EJ III, Nascarella MA (2011) Evidence for hormesis in mutagenicity dose-response relationships. Mutat Res Genet Toxicol Environ Mutagen 726(2):91-97

18. Cedergreen N, Hansen NKK, Arentoft BW (2016) The influence of nitrogen and phosphorous status on glyphosate hormesis in Lemna minor and Hordeum vulgare. Eur J Agron 73:107-117
19. Chen XD, Seo M, Stelinski LL (2017) Behavioral and hormetic effects of the butenolide insecticide, flupyradifurone, on Asian citrus psyllid, Diaphorina citri. Crop Prot 98:102-107

20. Christiani DC, Zhou W (2002) Hormesis: the new approach in risk assessment? Hum Exp Toxicol 21(7):399-400

21. de Vasconcelos EC, Dalke CR, de Oliveira CMR (2017) Influence of select antibiotics on Vibrio fischeri and Desmodesmus subspicatus at mg/L concentrations. Environ Manage 60(1):157-164

22. Deng Y, Beadham I, Wu J, Chen X-D, Hu L, Gu J (2015) Chronic effects of the ionic liquid C(4)mim Cl towards the microalga Scenedesmus quadricauda. Environ Pollut 204:248-255

23. Dohar JE (2003) Evolution of management approaches for otitis externa. Pediatr Infect Dis J 22(4):299-308

24. Evans ME, Feola DJ, Rapp RP (1999) Polymyxin B sulfate and colistin: old antibiotics for emerging multiresistant gram-negative bacteria. Ann Pharmacother 33(9):960-967

25. Fan Y, Liu S-S, Qu R, Li K, Liu H-L (2017) Polymyxin B sulfate inducing timedependent antagonism of the mixtures of pesticide, ionic liquids, and antibiotics to Vibrio qinghaiensis sp.-Q67. RSC Adv 7(10):6080-6088

26. Frey AH (1993) Electromagnetic-field interactions with biological-systems. FASEB J 7(2):272-281

27. Ge HL, Liu SS, Zhu XW, Liu HL, Wang LJ (2011) Predicting hormetic effects of ionic liquid mixtures on luciferase activity using the concentration addition model. Environ Sci Technol 45(4):1623-1629

28. Guan X, Jiang X, Qiao J, Zhou G (2015) Decomplexation and subsequent reductive removal of EDTA-chelated Cu-II by zero-valent iron coupled with a weak magnetic field: performances and mechanisms. J Hazard Mater 300:688-694

29. Hazeem LJ, Bououdina M, Dewailly E, Slomianny C, Barras A, Coffinier Y, Szunerits S, Boukherroub R (2017) Toxicity effect of graphene oxide on growth and photosynthetic pigment of the marine alga Picochlorum sp. during different growth stages. Environ Sci Pollut Res 24(4):4144-4152

30. Izmaylov DM, Obukhova LK, Konradov AA (2005) Correlations of lifespan variation parameters in 128 successive generations of Drosophila melanogaster with changes in atmospheric pressure and geomagnetic activity. Int J Biometeorol 49(5):337-344

31. Jiang X, Qiao J, Lo IMC, Wang L, Guan X, Lu Z, Zhou G, Xu C (2015) Enhanced paramagnetic $\mathrm{C} u 2^{+}$ions removal by coupling a weak magnetic field with zero valent iron. J Hazard Mater 283:880-887

32. Kanel SR, Manning B, Charlet L, Choi H (2005) Removal of arsenic(III) from groundwater by nanoscale zero-valent iron. Environ Sci Technol 39(5):1291-1298

33. Krylov VV (2010) Effects of electromagnetic fields on parthenogenic eggs of Daphnia magna Straus. Ecotoxicol Environ Saf 73(1):62-66

34. Lepper PM, Held TK, Schneider EM, Bolke E, Gerlach H, Trautmann M (2002) Clinical implications of antibiotic-induced endotoxin release in septic shock. Intensive Care Med 28(7):824-833

35. Li J, Bao H, Xiong X, Sun Y, Guan X (2015) Effective Sb(V) immobilization from water by zero-valent iron with weak magnetic field. Sep Purif Technol 151:276-283

36. LiT, Liu S-S, Ru R, Liu H-L (2017) Global concentration additivity and prediction of the mixture toxicities: taking nitrobenzene derivatives as an example. Ecotoxicol Environ Saf 144:475-481

37. Liang L, Sun W, Guan X, Huang Y, Choi W, Bao H, Li L, Jiang Z (2014) Weak magnetic field significantly enhances selenite removal kinetics by zero valent iron. Water Res 49:371-380

38. Litovitz TA, Krause D, Montrose CJ, Mullins JM (1994) Temporally incoherent magnetic-fields mitigate the response of biological-systems to temporally coherent magnetic-fields. Bioelectromagnetics 15(5):399-409

39. Litter MI, Morgada ME, Bundschuh J (2010) Possible treatments for arsenic removal in Latin American waters for human consumption. Environ Pollut 158(5):1105-1118

40. Liu L, Liu S, Yu M, Chen F (2015) Application of the combination index integrated with confidence intervals to study the toxicological interactions of antibiotics and pesticides in Vibrio qinghaiensis sp.-Q67. Environ Toxicol Pharmacol 39(1):447-456

41. Liu S-S, Song X-Q, Liu H-L, Zhang Y-H, Zhang J (2009) Combined photobacterium toxicity of herbicide mixtures containing one insecticide. Chemosphere 75(3):381-388

42. Liu S, Zhang J, Zhang Y, Qin L (2012) APTox: assessment and prediction on toxicity of chemical mixtures. Acta Chim Sin 70(14):1511 (in Chinese) 
43. Long XY, Ye J, Zhao D, Zhang SJ (2015) Magnetogenetics: remote noninvasive magnetic activation of neuronal activity with a magnetoreceptor. Sci Bull 60(24):2107-2119

44. Mark G. Papich DVM, M., DACVCP (2016) Polymyxin B sulfate. Elsevier, Amsterdam

45. Messiha HL, Wongnate T, Chaiyen P, Jones AR, Scrutton NS (2015) Magnetic field effects as a result of the radical pair mechanism are unlikely in redox enzymes. J R Soc Interface 12(103):20141155

46. Mevissen M, Haussler M, Loscher W (1999) Alterations in ornithine decarboxylase activity in the rat mammary gland after different periods of $50 \mathrm{~Hz}$ magnetic field exposure. Bioelectromagnetics 20(6):338-346

47. Nancharaiah YV, Francis AJ (2015) Hormetic effect of ionic liquid 1-ethyl3-methylimidazolium acetate on bacteria. Chemosphere 128:178-183

48. Negro CL, Senkman LE, Marino F, Lorenzatti E, Collins P (2014) Effects of chlorpyrifos and endosulfan on different life stages of the freshwater burrowing crab Zilchiopsis collastinensis P.: protective role of chorion. Bull Environ Contam Toxicol 92(6):625-630

49. Ng W-L, Bassler BL (2009) Bacterial quorum-sensing network architectures. Ann Rev Genet 43:197-222

50. Nogueira LS, Bianchini A, Smith S, Jorge MB, Diamond RL, Wood CM (2017) Physiological effects of five different marine natural organic matters (NOMs) and three different metals ( $\mathrm{Cu}, \mathrm{Pb}, \mathrm{Zn})$ on early life stages of the blue mussel (Mytilus galloprovincialis). Peer J 5:e3141

51. Novikov W, Shvezov YP, Fesenko EE, Novikova NI (1997) Molecular mechanisms of biological action of weak magnetic fields. 1. Stability of chromatin from Ehrlich ascite carcinoma and mouse brain cells towards DNAase 1 under combined action of weak constant and alternating lowfrequency magnetic fields adjusted to the cyclotronic resonance of ions of polar amino acids. Biofizika 42(3):733-737

52. Plawiak-Mowna A, Krawczyk A (2011) Remote monitoring of pacemakers and implantable cardioverter-defibrillators, safety aspects and study in cardiac implant patients on EMF exposure. Przeglad Elektrotechniczny 87(12B):137-140

53. Qin L-T, Liu S-S, Liu H-L, Zhang Y-H (2010) Support vector regression and least squares support vector regression for hormetic dose-response curves fitting. Chemosphere 78(3):327-334

54. Qin SY, Yin H, Yang CL, Dou YF, Liu ZM, Zhang P, Yu H, Huang YL, Feng J, Hao JF, Hao J, Deng LZ, Yan XY, Dong XL, Zhao ZX, Jiang TJ, Wang HW, Luo SJ, Xie C (2016) A magnetic protein biocompass. Nat Mater 15(2):217-226

55. Qu R, Liu S-S, Chen F, Li K (2016) Complex toxicological interaction between ionic liquids and pesticides to Vibrio qinghaiensis sp.-Q67. RSC Adv 6(25):21012-21018

56. Qu R, Liu S-S, LiT, Liu H-L (2019) Using an interpolation-based method (IDVequ) to predict the combined toxicities of hormetic ionic liquids. Chemosphere 217:669-679

57. Radak Z, Ishihara K, Tekus E, Varga C, Posa A, Balogh L, Boldogh I, Koltai E (2017) Exercise, oxidants, and antioxidants change the shape of the bellshaped hormesis curve. Redox Biol 12:285-290

58. Rapis EG (1997) Magnetic sensitivity of protein. Tech Phys Lett 23(4):263-267

59. Saber M, Hejazi MJ, Hassan SA (2004) Effects of azadirachtin/neemazal on different stages and adult life table parameters of Trichogramma cacoeciae (Hymenoptera:Trichogrammatidae). J Econ Entomol 97(3):905-910

60. Stebbing ARD (1998) A theory for growth hormesis. Mutat Res Fundam Mol Mech Mutagen 403(1-2):249-258

61. Steblenko LP, Nigelska Al, Naumenko SN, Kuryliuk AN, Kobzar YL, Yakunov AV, Krit AN, Kalinichenko DV (2017) Effect of a weak magnetic field and low-energy $\mathrm{X}$-rays on the evolution of Saccharomyces cerevisiae yeast suspension. J Surf Investig 11(1):280-284

62. Sugie C, Shibamoto Y, Hashimoto S, Tsuchiya T, Matsuo M, Kawai T, Iwata $H$ (2016) Biological effects of a radiation hormesis sheet emitting very low-dose-rate gamma rays. Int J Radiat Oncol Biol Phys 96(2):E561-E562

63. Sun Y, Guan X, Wang J, Meng X, Xu C, Zhou G (2014) Effect of weak magnetic field on arsenate and arsenite removal from water by zerovalent iron: an XAFS investigation. Environ Sci Technol 48(12):6850-6858

64. Sun Y, Hu Y, Huang T, Li J, Qin H, Guan X (2017) Combined effect of weak magnetic fields and anions on arsenite sequestration by zerovalent iron: kinetics and mechanisms. Environ Sci Technol 57(7):3742-3750

65. Sutherland RM, Marton JP, Macdonald JCF, Howell RL (1978) Effect of weak magnetic-fields on growth of cells in tissue-culture. Physiol Chem Phys 10(2):125-131
66. Tarasevich YY (2004) Mechanisms and models of the dehydration selforganization in biological fluids. Phys Uspekhi 47(7):717-728

67. Wang M, Liu S, Chen F (2014) Predicting the time-dependent toxicities of three triazine herbicide mixtures to V. qinghaiensis sp. Q67 using the extended concentration addition model. Acta Chim Sin 72(1):56 (in Chinese)

68. Wang Z, Liu X, Ni S-Q, Zhang J, Zhang X, Ahmad HA, Gao B (2017) Weak magnetic field: a powerful strategy to enhance partial nitrification. Water Res 120:190-198

69. Wang Z-J, Liu S-S, Ru R (2018) JSFit: a method for the fitting and prediction of J-and S-shaped concentration-response curves. RSC Adv 8(12):6572-6580

70. Xu C, Zhang B, Zhu L, Lin S, Sun X, Jiang Z, Tratnyek PG (2016) Sequestration of antimonite by zerovalent iron: using weak magnetic field effects to enhance performance and characterize reaction mechanisms. Environ Sci Technol 50(3):1483-1491

71. Xu J, Pan W, Zhang Y, Li Y, Wan G, Chen F, Sword GA, Pan W (2017) Behavioral evidence for a magnetic sense in the oriental armyworm, Mythimna separata. Biol Open 6(3):340-347

72. Xu Y, Hou M, Ruan J, Qu M, Sun H, Xu J, Zhou S (2014) Effect of magnetic field on surface properties of Bacillus cereus CrA and its extracellular polymeric substances (EPS). J Adhes Sci Technol 28(21):2196-2208

73. Xu Y-Q, Liu S-S, Fan Y, Li K (2018) Toxicological interaction of multi-component mixtures to Vibrio qinghaiensis sp.-Q67 induced by at least three components. Sci Tot Environ 635:432-442

74. Xu Y-Q, Liu S-S, Wang Z-J, Li K, Qu R (2018) Commercial personal care product mixtures exhibit hormetic concentration-responses to Vibrio qinghaiensis sp.-Q67. Ecotoxicol Environ Saf 162:304-311

75. Xu Y-Q, Liu S-S, Li K, Wang Z-J, Xiao Q-F (2019) Polyethylene glycol 400 significantly enhances the stimulation of 2-phenoxyethanol on Vibrio qinghaiensis sp.-Q67 bioluminescence. Ecotoxicol Environ Saf 171:240-246

76. Yakhno TA, Yakhno VG, Sanin AG, Sanina OA, Pelyushenko AS (2004) Protein and salt: spatiotemporal dynamics of events in a drying drop. Tech Phys 49(8):1055-1063

77. Yang H, Wang Y, Yang J, Wu T (2016) Dosimetry of electromagnetic field exposure of an active armlet and its electromagnetic interference to the cardiac pacemakers using adult, child and infant models. Electromagn Biol Med 35(2):120-125

78. Yu M, Liu S, Wang M, Chen F, Tang H (2014) Mixture toxicities of three pesticides having different time-toxicity profiles. Chin J Chem 32(6):545-552

79. Yan W, Tang F, He L, Chen J, Xin Z, Sun X, Huang Z, Kuai L (2017) Effect of gamma ray radiation on the luminous intensity of Vibrio qinghaiensis sp. Q67. Nucl Tech 40(12):120502 (in Chinese)

80. Zhang J, Ding C, Ren L, Zhou Y, Shang P (2014) The effects of static magnetic fields on bone. Prog Biophys Mol Biol 114(3):146-152

81. Zhang J, Liu S-S, Yu Z-Y, Liu H-L, Zhang J (2013) The time-dependent hormetic effects of 1-alkyl-3-methylimidazolium chloride and their mixtures on Vibrio qinghaiensis sp.-Q67. J Hazard Mater 258:70-76

82. Zhang L, Jiang J, Ning P, Chen L, Zhu Y (2004) The hormesis of blood samples irradiated in vitro by $\mathrm{X}$-rays to low doses. J Radiat Res Radiat Process 22(5):315-317

83. Zhao W, Hu N, Ding D, Long D, He S, Li L, Hu M, Feng Y (2017) Study on toxicity of low dose gamma radiation to Qinghai Vibrio Q67. Radiat Prot 37(2):138-144 (in Chinese)

84. Zheng Q-F, Ju Z, Liu S-S (2019) Combined toxicity of dichlorvos and its metabolites to Vibrio qinghaiensis sp-Q67 and Caenorhabditis elegans. Acta Chim Sin 77(10):1008-1016 (in Chinese)

85. Zhu C-J, Peng Y, Tong Z-H, Lu L-Y, Cui Y-H, Yu H-Q (2016) Hormetic effect and mechanism of imidazolium-based ionic liquids on the nematode Caenorhabditis elegans. Chemosphere 157:65-70

\section{Publisher's Note}

Springer Nature remains neutral with regard to jurisdictional claims in published maps and institutional affiliations. 